\title{
A Comparison of Color Correction Algorithms for Endoscopic Cameras
}

\author{
Ioannis Constantinou, Marios Neofytou, Vasilis Tanos, Marios Pattichis, \\ Christodoulos Christodoulou and Constantinos Pattichis
}

\begin{abstract}
Quantitative color tissue analysis in endoscopy examinations requires color standardization procedures to be applied, so as to enable compatibility among computer aided diagnosis application from different endoscopy labs. The objective of this study was to examine the usefulness of different color correction algorithms (thus facilitating color standardization), evaluated on four different endoscopy cameras. The following five color correction algorithms were investigated: two gamma correction based algorithms (the classical and a modified one), and three $\left(2^{\text {nd }}, 3^{\text {rd }}\right.$, and $4^{\text {th }}$ order) polynomial based correction algorithms. The above algorithms were applied to four different endoscopy cameras: (a) Circon, (b) Karl-Stortz, (c) Olympus, and (d) Snowden-Pencer. The color correction algorithms and the endoscopic cameras evaluation, was carried out using the testing color palette ( 24 colors of known digital values) provided by the Edmund Industrial Optics Company. In summary, we have that: (a) the modified gamma correction algorithm gave significantly smaller mean square error compared to the other four algorithms, and (b) the smallest mean square error was obtained for the Circon camera. Future work will focus on evaluating the proposed color correction algorithm in different endoscopy clinics and compare their tissue characterization results.
\end{abstract}

\section{INTRODUCTION}

In recent years, endoscopy, colonoscopy, gatroendoscopy, and laryngoscopy examinations use medical cameras to monitor human organs with minimum complications. These methods are the most popular ones, compared with classical open surgery procedures which are used in special conditions due to the complications that are associated with [1]-[4].

Quantitative color tissue analysis in endoscopy examinations requires color standardization procedures to be applied; enabling computer aided diagnosis, as well as monitoring the sensitivity and specificity performance of different endoscopy labs. The objective of this study was to examine the usefulness of different color correction algorithms (thus facilitating color standardization), evaluated on four different endoscopy cameras.

These will support the physician opinion and will help her/him to increase the accuracy of the diagnosis providing

Manuscript received July 30, 2013

Ioannis Constantinou, Marios Neofytou and Constantinos Pattichis are with the Department of Computer Science at the University of Cyprus. ikonst01i@ucy.ac.cy, mneoph@ucy.ac.cy, pattichii@ucy.ac.cy

Vasilis Tanos is with the Aretaeio Medical Center, Nicosia, Cyprus.v.tanosearetaeio.com

Marios Pattichis is with the Department of Electrical and Computer Engineering, University of New Mexico, Albuquerque, NM 87131 USA. pattichis lece.umn.edu

Christodoulos Christodoulou is with the Department of Computer Science at the University of Cyprus. cschr2eucy.ac.cy better quality videos/images.

It was demonstrated by our group that gamma color correction is a necessary pre-processing component for the development of a Computer Aided Diagnostic (CAD) system able to differentiate between normal and abnormal endometrial tissue in the early stages of gynaecological cancer based on color texture analysis in hysteroscopy imaging [5]-[7]. The need of standardization efforts for reporting endoscopy examinations including color standardization were proposed in [8]. Moreover, the usefulness of gamma color correction was clearly demonstrated in microscopy imaging as well [9].

Additional algorithms for color standardization include the use of polynomial correction fitting, recently used for regular cameras as well [10].

The structure of this paper is as follows. Section II presents the methodology. Section III the results, and Section IV the concluding remarks.

\section{METHODOLOGY}

\section{A. Recording of Endoscopic Video}

The following endoscopy cameras were used: Circon [11], Karl-Storz [12], Olympus [13], and Snowden-Pencer [14].

\section{B. Recording of Testing Targets}

The testing color palette provided by Edmund Industrial Optics Company [15], with known color distribution was used in this set of experiments (see Figure 1, and Table I). The general purpose of a test pattern is to determine the true color balance or optical density of any color system. It is an industry standard that provides a non-subjective comparison with a test pattern of 24 carefully prepared colored squares (see Figure 1). Each square in the pattern represents a natural color like the human skin, foliage, blue sky, etc.

Testing images were captured in the surgery room with natural daylight illumination (D65) and focusing based on the experience of the physician, using the four cameras under investigation. Following the above procedure, we captured and saved the video (AVI format) of the testing palette using the VCE-PRO frame grabber [16], (24 bits color at 25 frames per second) and then extracted uncompressed TIFF images of the 24 color squares, 64X64 pixels. The corresponding targets were digitally generated based on the data given by the Edmund Optics Company [15], as the ground truth of the experiment (see Figure 1). RGB values for some of the testing targets provided by the manufacturer are given in Table I. 


\section{Color correction algorithms}

1) Classical Gamma Correction: The classical gamma correction algorithm based on the following equations was used in [17]:

$$
\begin{aligned}
& R_{\text {out }}=\alpha_{R} R_{i n}^{\gamma_{R}}+b_{R} \\
& G_{\text {out }}=\alpha_{G} G_{i n}^{\gamma_{G}}+b_{G} \\
& B_{\text {out }}=\alpha_{B} B_{\text {in }}^{\gamma_{B}}+b_{B} .
\end{aligned}
$$

The $R_{i n}, G_{i n}$ and $B_{\text {in }}$ denote the original Red, Green and Blue color signals and $R_{\text {out }}, G_{\text {out }}$ and $B_{\text {out }}$ denote the corrected output color signals. The computation of the parameters was based on the non-linear least squares algorithm (see 1sqnonlin function in MATLAB [18]]). We estimate the gamma and offset values in each channel, $R, G, B$ and $b_{R}, b_{G}, b_{B}$ respectively (see eq. 1).

TABLE I

R, G, AND B VALUES OF COLOR PALLET FROM THE EDMUND INDUSTRIAL OPTICS COMPANY

\begin{tabular}{cccc}
\hline Color & $\mathrm{R}$ & $\mathrm{G}$ & $\mathrm{B}$ \\
\hline \hline Black & 0 & 0 & 0 \\
White & 255 & 255 & 255 \\
Red & 203 & 0 & 0 \\
Green & 64 & 173 & 38 \\
Blue & 0 & 0 & 142 \\
Dark skin & 94 & 28 & 13 \\
Light skin & 241 & 149 & 108 \\
Blue sky & 97 & 119 & 171 \\
Foliage & 90 & 103 & 39 \\
Blue flower & 164 & 131 & 196 \\
Orange & 255 & 116 & 21 \\
Magenta & 207 & 3 & 124 \\
\hline \hline
\end{tabular}

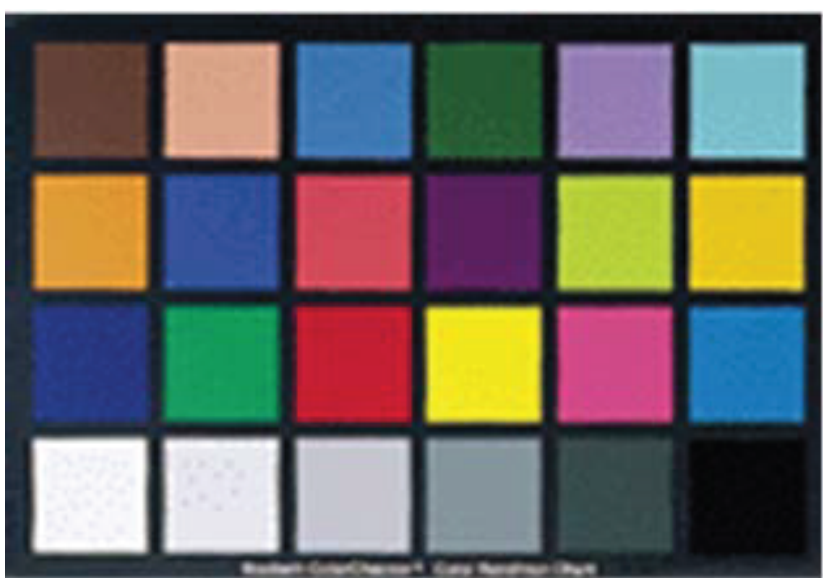

Fig. 1. The testing targets of the color palette with known color distribution from the Edmund Industrial Optics Company [15]

2) Gamma Correction algorithm: This model is based on the following equations:

$$
\left[\begin{array}{c}
R_{\text {out }} \\
G_{\text {out }} \\
B_{\text {out }}
\end{array}\right]=\left[\begin{array}{lll}
a_{11} & a_{12} & a_{13} \\
a_{21} & a_{22} & a_{23} \\
a_{31} & a_{32} & a_{33}
\end{array}\right]\left[\begin{array}{c}
R_{\text {in }} \\
G_{\text {in }} \\
B_{\text {in }}
\end{array}\right]+\left[\begin{array}{l}
k_{1} \\
k_{2} \\
k_{3}
\end{array}\right]=
$$

$$
=A\left[\begin{array}{l}
R_{\text {in }} \\
G_{\text {in }} \\
B_{\text {in }}
\end{array}\right]+k
$$

$$
\begin{aligned}
& R_{\text {out }}=255\left(R_{\text {out }^{\prime}} / 255\right)^{\gamma_{R}} \\
& G_{\text {out }}=255\left(G_{\text {out }^{\prime}} / 255\right)^{\gamma_{G}} \\
& B_{\text {out }}=255\left(B_{\text {out }^{\prime}} / 255\right)^{\gamma_{B}} .
\end{aligned}
$$

The $\left[\begin{array}{lll}R_{\text {out }} & G_{\text {out }} & B_{\text {out }}{ }^{\prime}\end{array}\right]^{T}$ denote the original input Red, Green, and Blue components of the target image (see eq. 2 , 3). The $\left[\begin{array}{lll}R_{\text {out }} & G_{\text {out }} & B_{\text {out }}\end{array}\right]^{T}$ denote the output (corrected) RGB components of the target image. The output signal was multiplied, using a linear matrix $A$ and a constant offset matrix $k$. The non-linear gamma model can be approached using (3). The computation of the parameters was based on the non-linear least squares algorithm (see lsqnonlin function in MATLAB [18]). We estimate matrices $A, k$ and the gamma values in each channel, $R, G, B$ and the result is the correction of the output signal.

3) Polynomial $2^{\text {nd }}, 3^{\text {rd }}$, and $4^{\text {th }}$ order Correction: The polynomial $2^{\text {nd }}, 3^{\text {rd }}$, and $4^{\text {th }}$ order correction algorithms were based on the following equation (given for the case of $4^{\text {th }}$ order) [10]:

$$
\begin{gathered}
R_{\text {out }}=\alpha_{R_{0}}+\alpha_{R_{1}} R_{\text {in }}+\alpha_{R_{2}} R_{\text {in }}^{2}+\alpha_{R_{3}} R_{\text {in }}^{3}+\alpha_{R_{4}} R_{\text {in }}^{4} \\
G_{\text {out }}=\alpha_{G_{0}}+\alpha_{G_{1}} G_{i n}+\alpha_{G_{2}} G_{i n}^{2}+\alpha_{G_{3}} G_{i n}^{3}+\alpha_{G_{4}} G_{i n}^{4} \\
B_{\text {out }}=\alpha_{B_{0}}+\alpha_{B_{1}} B_{\text {in }}+\alpha_{B_{2}} B_{\text {in }}^{2}+\alpha_{B_{3}} B_{\text {in }}^{3}+\alpha_{B_{4}} B_{\text {in }}^{4}
\end{gathered}
$$

The $R_{i n}, G_{i n}$ and $B_{\text {in }}$ denote the original Red, Green and Blue color signals and $R_{\text {out }}, G_{\text {out }}$ and $B_{\text {out }}$ denote the corrected output color signals. The computation of the terms for each channel was based on the non-linear least squares algorithm (see lsqnonlin function in MATLAB [18])

\section{Parameter Estimation Using Cross Validation}

Two different experiments were carried out. In experiment 1 only one set of testing target videos were recorded for the 4 different endoscopic cameras. No cross validation was carried out, and the color correction was applied on the same set of images the correction parameters were derived.

In experiment 2, 5 different sets of color targets were extracted from the captured videos, and five different runs of the color correction algorithms were carried out using the leave one out method. Thus in each run, the training (i.e. extracting of the color correction parameters) was carried out on the 4 set of targets, and the evaluation (i.e. application of the color correction parameters) on the remaining one.

\section{RESULTS}

\section{A. Experiment I}

Table II tabulates the mean square error (MSE) for 5 color correction algorithms for the 4 cameras under study. The gamma correction algorithm gave the smallest MSE values. The Circon camera gave also the smallest MSE values, followed by the Karl-Stortz camera. 
TABLE II

MEAN SQUARE ERROR FOR EACH COLOR CORRECTION ALGORITHM FOR 4 DIFFERENT ENDOSCOPIC CAMERAS (SEE III.A EXPERIMENT 1)

\begin{tabular}{|c|c|c|c|c|c|c|c|c|c|c|c|c|c|c|c|c|}
\hline \multirow{2}{*}{$\begin{array}{c}\text { COLOR CORRECTION } \\
\text { ALGORITHM }\end{array}$} & \multicolumn{4}{|c|}{ Circon } & \multicolumn{4}{|c|}{ Karl-Storz } & \multicolumn{4}{|c|}{ Olympus } & \multicolumn{4}{|c|}{ Snowden-Pencer } \\
\hline & $\mathrm{R}$ & $\mathrm{G}$ & $\mathrm{B}$ & $\sum$ RGB & $\mathrm{R}$ & $\mathrm{G}$ & $\mathrm{B}$ & $\sum \mathrm{RGB}$ & $\mathrm{R}$ & $\mathrm{G}$ & $\mathrm{B}$ & $\sum \mathrm{RGB}$ & $\mathrm{R}$ & $\mathrm{G}$ & $\mathrm{B}$ & $\sum \mathrm{RGB}$ \\
\hline Gamma Correction & 40 & 58 & 93 & 191 & 110 & 62 & 35 & 207 & 203 & 31 & 62 & 296 & 250 & 135 & 839 & 1224 \\
\hline Polynom & 416 & 53 & 314 & 783 & 605 & 100 & 467 & 1172 & 468 & 31 & 393 & 892 & 1421 & 165 & 1251 & 2837 \\
\hline Polynomial 4th order & 402 & 48 & 290 & 740 & 1109 & 92 & 477 & 1678 & 475 & 32 & 390 & 897 & 1421 & 159 & 1251 & 2831 \\
\hline
\end{tabular}

R, G, B represent the MSE for the Red, Green, and Blue channels respectively, whereas $\sum$ RGB represents their sum.

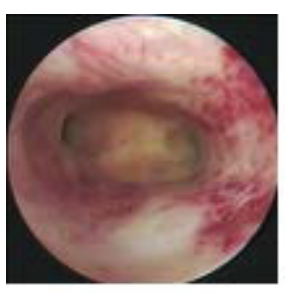

a

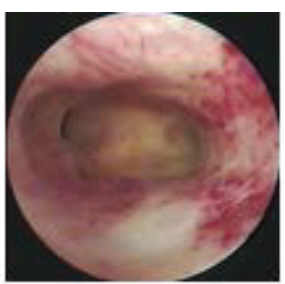

b
Fig. 2. Endometrium images (a) before and (b) after gamma correction algorithm.

\section{B. Experiment II}

Table III tabulates the results of the mean of the MSE for 5 runs for the 5 color correction algorithms. The mean of the MSE for the 5 runs for the evaluation set was computed for each color correction algorithm for each channel. In this experiment, only the Circon endoscopic camera was used (i.e. the one that gave the smallest MSE in Experiment 1). Again, the gamma correction algorithm gave the smallest MSE values. An Example of the Application of Color Correction on Hysteroscopy Imaging Figure 2 gives an example of an uncorrected and the corresponding corrected hysteroscopy images of the endometrium. It is shown that the corrected image is brighter, and based on physician opinion it is of better quality, and can support her/his diagnosis easier.

TABLE III

MEAN SQUARE ERROR FOR EACH COLOR CORRECTION ALGORITHM FOR THE CIRCON ENDOSCOPIC CAMERA (SEE III.B EXPERIMENT 2)

\begin{tabular}{ccccc}
\hline \hline COLOR CORRECTION & \multicolumn{4}{c}{ Circon } \\
\cline { 2 - 5 } ALGORITHM & $\mathrm{R}$ & $\mathrm{G}$ & $\mathrm{B}$ & $\sum \mathrm{RGB}$ \\
\hline \hline Classical Correction & 444 & 375 & 940 & 1759 \\
Gamma Correction & 147 & 73 & 181 & 401 \\
Polynomial 2nd order & 449 & 76 & 542 & 1067 \\
Polynomial 3rd order & 394 & 76 & 510 & 980 \\
Polynomial 4th order & 551 & 868 & 518 & 1937 \\
\hline \hline
\end{tabular}

\section{CONCLUDING REMARKS}

Quantitative color tissue analysis in endoscopy examinations requires color standardization procedures to be applied, enabling computer aided. The objective of this study was to examine the usefulness of different color correction algorithms (thus facilitating color standardization), evaluated on four different endoscopy cameras. The following five color correction algorithms were investigated: two gamma correction based algorithms (the classical and a modified one), and three $\left(2^{\text {nd }}, 3^{\text {rd }}\right.$, and $4^{\text {th }}$ order) polynomial based correction algorithms. These algorithms were applied on four different endoscopy cameras: (a) Circon, (b) Karl-Stortz, (c) Olympus, and (d) Snowden-Pencer. The color correction algorithms and the endoscopic cameras evaluation, was carried out using the testing color palette ( 24 colors of known digital values) provided by the Edmund Industrial Optics Company. It was shown that: (a) the modified gamma correction algorithm gave significantly smaller mean square error compared to the other four algorithms, and (b) the smallest mean square error was obtained for the Circon camera. Thus, the color correction algorithm can be used to standardize endoscopy images, given that to the best of our knowledge, there are no guidelines for the color calibration of the endoscopy cameras [19]-[24].

Furthermore, these findings were applied to hysteroscopy imaging for differentiating between normal and abnormal tissue of the endometrium with very promising results. More specifically, the Circon endoscopy camera was used, and the gamma color correction algorithm. Texture analysis on the manually segmented regions of interest (ROIs) demonstrated that normal ROIs have higher gray level, and lower variance and contrast when compared to abnormal ROIs [5]. Homogeneity was slightly lower, and entropy slightly higher of the abnormal ROIs when compared to the normal ones.

Future work will focus on evaluating the proposed color correction algorithm in different endoscopy clinics using different equipment and compare their tissue characterization results. Furthermore, we hope that the proposed methodology can also be applied to other endoscopic modalities such as colonoscopy and gastro endoscopy.

\section{REFERENCES}

[1] J.A. Fayez, M. F. Vogel, "Comparison of different treatment methods of endometriomas by laparoscopy”, Obstet. Gynecol., Vol. 78, pp. 660-665, 1991.

[2] J.F. Willem, V.B. Corla et al., "Complications of hysteroscopy: A Prospective, Multicenter Study”, Obstet. Gynecol., Vol. 92, issue 2, pp. 266-270, 2000. 
[3] P.M. Tjoa, M.S. Krishnan, "Feature Extraction for the Analysis of Colon Status from the Endoscopic Images", BioMedical Engineering OnLine, Apr. 2003. [http://www.biomedical-engineeringonline.com/content/2/1/9].

[4] J. Ilgner, C. Palm, A. Schutz, K. Spitzer, M. Westhofen, T. Lehmann, "Colour Texture Analysis for Quantitative Laryngoscopy", Acta Otolaryngol., vol. 123, pp. 730-734, 2003.

[5] M.S. Neophytou, C.S. Pattichis, M.S. Pattichis, V. Tanos, E.C. Kyriacou, D. Koutsouris, "A Standardised Protocol for Texture Feature Analysis of Endoscopic Images in Gynaecological Cancer", BioMedical Engineering OnLine, available at http://www.biomedical-engineeringonline.com/start.asp, Vol. 6, 2007.

[6] I.P. Constantinou, C.A. Koumourou, M.S. Neofytou, V. Tanos, C.S Pattichis, E.C. Kyriakou, "An integrated CAD system facilitating the endometrial cancer diagnosi"s, 9th International Conference on Information Technology and Applications in Biomedicine, November 5-7, Larnaca, Cyprus, 5 pages, 2009.

[7] M.S. Neofytou, V. Tanos, M.S. Pattichis, C.S. Pattichis, E.C. Kyriacou, S. Pavlopoulos, "Color Based Texture - Classification of Hysteroscopy Images of the Endometrium", 29th Annual International Conference of the IEEE Engineering in Medicine and Biology Society, August 23-27, Lyon, France, 2007.

[8] H. Yokoi, M.A. Fujino, "Activities for Endoscopy Information Systems Standardization in Japan", 28th Annual International conference of the IEEE engineering in Medicine and Biology Society, September, New York, USA, pp. 5667-5670, 2006.

[9] Y. Yukako, "Color standardization and optimization in whole slide imaging", Diagn Pathol 6.Suppl 1 (2011): S15.

[10] C. Vien, S. Westland, D. Connah, C. Ripamonti, "A comparative study of the characterisation of colour cameras by means of neural networks and polynomial transforms". Coloration technology, Vol. 120, pp. 19-25, 2004.

[11] The company Circon [http://www.circoncorp.com]

[12] The company Karl Storz: http://www.karlstorz.com/

[13] The Olympus Corporation: [http://www.olympus-global.com]

[14] The Company CAREFusion: [http://www.carefusion.com]

[15] Web link: http://www.edmundoptics.com/

[16] Web link: http://www.imperx.com/

[17] M.D. Grossberg, S.K. Nayar, "Modeling the space of camera response functions", IEEE Trans Pattern Anal, Mach, Intell., vol. 26, issue 10, pp. 1272-1282, 2004.

[18] Web link: http://www.mathworks.com

[19] S. Sheraizin, V. Sheraizin, "Endoscopy Imaging Intelligent Contrast Improvement", 27th Annual International conference of the IEEE engineering in Medicine and Biology Society, September 1-4, Shanghai, China, 4 pages, 2005.

[20] J. Scarcanski, W. Gaviao, S. Cunha, F. Joao, "Diagnostic Hysteroscopy Video Summarization and Browsing", 27th Annual International conference of the IEEE engineering in Medicine and Biology Society, September 1-4, Shanghai, China, 4 pages, 2005.

[21] X. Shunren, M. Weirong, W. Xiaoying, Z. Zanchao, "A content-Based retrieval system for Endoscopic Images", 27th Annual International conference of the IEEE engineering in Medicine and Biology Society, September 1-4, Shanghai, China, 4 pages, 2005.

[22] W. Gaviao, J. Scharcanski, "Content-Based Diagnostic Hysteroscopy Summaries for Video Browsing", Computer Graphics and Image Processing, SIBGRAPI 2005, 18th Brazilian Symposium on, vol., no., pp. 21- 28, 09-12 Oct. 2005.

[23] K.N. Plataniotis, A.N. Venetsanopoulos, "Color Image Processing and Applications", Springer Verlag. Berlin, August, 2000.

[24] Y. Vander Haeghen, J. Naeyaert, I. Lemahieu, W. Philips, "An Imaging System with Calibrated Color Image Acquisition for Use in Dermatology", IEEE Transactions on Medical Imaging, July 2000, vol. 19: no 